\title{
RICCI CURVATURE OF LAGRANGIAN SUBMANIFOLDS IN COMPLEX SPACE FORMS
}

\author{
TEODOR OPREA
}

Abstract. As we showed in [5] and [6], the basic inequalities, involving Riemannian invariants of a Lagrangian submanifold immersed in a complex space form, can be improved using optimization methods. Also in [1] is showed that the improved Chen's inequality from [5] is optimal. In this paper we find another proof for a Chen's inequality, regarding the Ricci curvature [2] and we improve this inequality in the Lagrangian case.

Mathematics subject classification (2010): 53C21, 53C24, 53C25, 49K35.

Keywords and phrases: Constrained maximum, Chen's inequality, Lagrangian submanifolds.

\section{REFERENCES}

[1] J. Bolton, L. VRancKen, Lagrangian submanifolds attaining equality in the improved Chen's inequality, Bull. Belg. Math. Soc., 14, 2 (2007), 311-315.

[2] B.Y. CHEN, Mean curvature shape operator of isometric immersions in real-space-forms, Glasgow Math. J., 38 (1996), 87-97.

[3] B.Y. CHEN, Relations between Ricci curvature shape operator for submanifolds with arbitrary codimensions, Glasgow Math. J., 41 (1999), 33-41.

[4] T. Oprea, Optimizations on Riemannian Submanifolds, Analele Univ. Buc., LIV, 1 (2005), 127-136.

[5] T. Oprea, Chen's inequality in the Lagrangian case, Colloq. Math., 108, 1 (2007), 163-169.

[6] T. Oprea, On a Riemannian invariant of Chen type, Rocky Mountain J. Math., 38, 2 (2008), 567-581.

[7] C. UdRIŞTE, Convex functions and optimization methods on Riemannian manifolds, Kluwer Academic Publishers Group, Dordrecht, 1994.

[8] C. UdrişTe, O. Dogaru, I ŢEvy, Extrema with nonholonomic constraints, Geometry Balkan Press, Bucharest, 2002. 